\title{
La calcifilassi nell'insufficienza renale cronica
}

\author{
Gioacchino Li Cavoli, Luisa Bono, Calogera Tortorici, Audenzia Manno, Roberta Benfante, Tancredi Vincenzo Li Cavoli, \\ Ugo Rotolo
}

UOC Nefrologia e Dialisi, ARNAS Civico-Benfratelli-Di Cristina, Palermo

\begin{abstract}
Calciphylaxis in chronic kidney disease
Purpose: Calciphylaxis or Calcific Uraemic Arteriolopathy (CUA) is a rare systemic disorder associated with endstage renal disease and carrying a very poor prognosis and high mortality. It is characterized by skin necrosis and gangrene secondary to thrombosis and calcification of dermo-epidermal arterioles. Pathogenetic mechanisms inducing calciphylaxis are for the most part unknown.

Methods: We report a case of calciphylaxis in a patient on long-term haemodialysis with severe secondary hyperparathyroidism. Despite therapies for hyperparathyroidism, the injury healed only after parathyroidectomy. Results: Calciphylaxis is an obliterative vasculopathy. The role of severe hyperparathyroidism in the pathogenesis of calciphylaxis is not well understood. A unique feature is the phenotypic differentiation of smooth muscle cells into osteoid-like cells. The vascular smooth muscle cells, exposed to high inorganic phosphate levels, could be transformed into osteoblast-like cells in patients undergoing haemodialysis with a poor control of phosphate intake.

Conclusions: The uraemic condition contributes to vascular smooth muscle cell apoptosis and differentiation into bone forming osteoblast-like cells resulting in medial calcification. Nowadays no effective treatments are available for the successful management of calciphylaxis.
\end{abstract}

Keywords: Bone metabolism, Calcific uraemic arteriolopathy, Calciphylaxis, Haemodialysis, Hyperparathyroidism

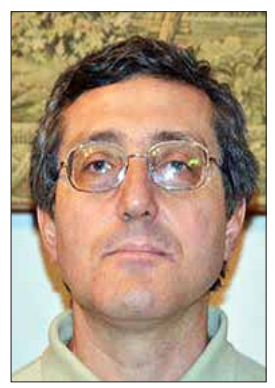

Gioacchino Li Cavoli

\section{Introduzione}

La calcifilassi o CUA è una rara patologia sistemica a preminente interessamento cutaneo. Essa rappresenta una grave complicanza della malattia renale cronica (MRC) in fase dialitica ed è gravata da un'elevata mortalità. Si caratterizza per una necrosi ischemica cutanea conseguente a fenomeni trombotici e alla calcificazione delle arteriole dermoepidermiche. Le calcificazioni vascolari sono un elemento fisiopatologico fondamentale della malattia cardiovascolare, principale causa di morte dei pazienti con malattia renale cronica (MRC). La calcifilassi è una patologia

Accepted: April 13, 2016

Published online: May 25, 2016

Indirizzo per la corrispondenza:

Dr. Gioacchino Li Cavoli

Via Francesco Cilea 43

90144 Palermo

gioacchinolicavoli@libero.it emergente in ambito nefrologico, dermatologico e cardiologico. I meccanismi patogenetici responsabili della CUA sono in massima parte sconosciuti e nessuna singola alterazione clinico-umorale è in grado di predire lo sviluppo di questa patologia (1-3). Presentiamo la nostra esperienza relativa a un caso di calcifilassi associato a iperparatiroidismo secondario alla malattia renale cronica.

\section{Presentazione del caso}

Un uomo di 52 anni, affetto da glomerulonefrite cronica, ha iniziato, nel 1999, il trattamento emodialitico. In questo periodo era in terapia con calcio carbonato $1 \mathrm{gr} \times 2 /$ die, idrossido di alluminio $400 \mathrm{mg} \times 2 /$ die e calcitriolo $0.25 \mathrm{mcg} /$ die; gli esami di laboratorio evidenziavano paratormone (PTH) 102 $\mathrm{pg} / \mathrm{mL}$, calcemia $9.8 \mathrm{mg} / \mathrm{dL}$, fosforemia $4.3 \mathrm{mg} / \mathrm{dL}$ e prodotto calcio-fosforo 42.1. Nel 2005, la terapia con calcitriolo è stata interrotta a causa di uno scarso controllo della fosforemia (6.8 $\mathrm{mg} / \mathrm{dL}$ ) e del prodotto calcio-fosforo (61.2); la calcemia, invece, si manteneva nella normalità, sebbene, in questo periodo, il PTH abbia raggiunto il valore di $299 \mathrm{pg} / \mathrm{mL}$; la terapia chelante del fosforo è stata pertanto modificata sostituendo il calcio carbonato con il sevelamer $800 \mathrm{mg} \times 3 /$ die; secondo le Linee Guida internazionali, è stata raccomandata la limitazione dell'apporto alimentare del fosforo (4). Nel 2006, il PTH 


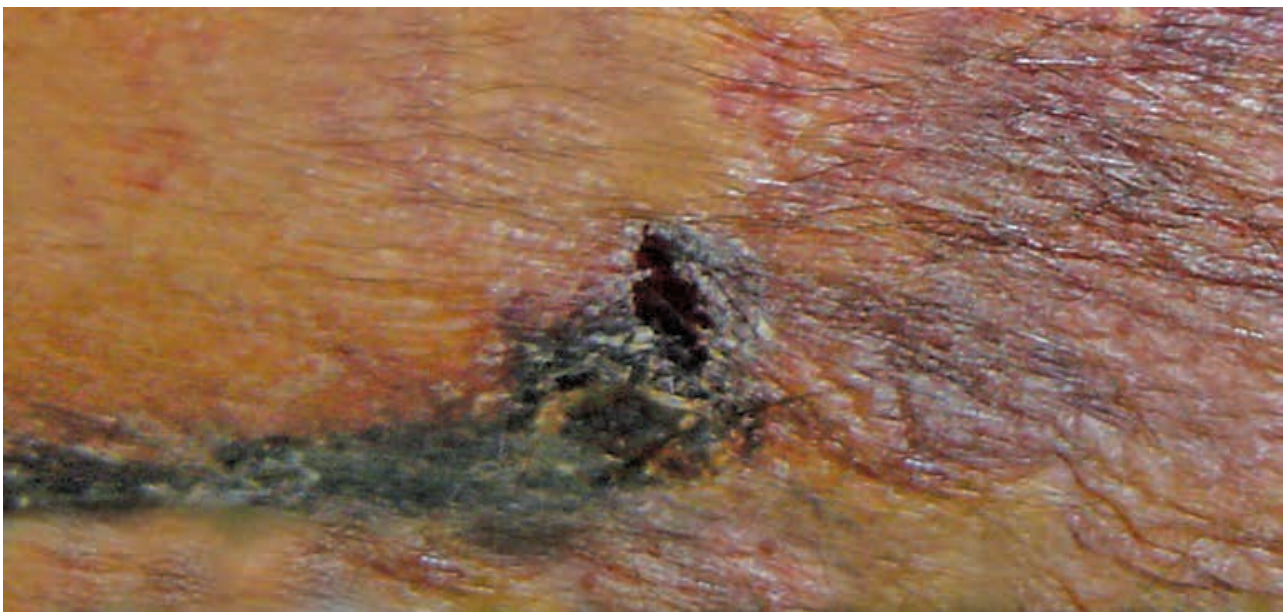

Fig. 1 - Lato dorsale della mano sinistra: lesione cutanea violacea con ulcerazione ed escara.

è aumentato a $828 \mathrm{pg} / \mathrm{mL}$ e il paziente ha iniziato una terapia con cinacalcet, dapprima $30 \mathrm{mg}$ e, quindi, $60 \mathrm{mg} / \mathrm{die}$, senza, tuttavia, alcun significativo miglioramento. Nel Gennaio del 2008, le indagini di laboratorio hanno rivelato una normale calcemia $(9.7 \mathrm{mg} / \mathrm{dL})$, una fosforemia pari a $5.6 \mathrm{mg} / \mathrm{dL}$ e un prodotto calcio-fosforo pari a 54.3, mentre il PTH è aumentato fino a $1421 \mathrm{pg} / \mathrm{mL}$; al cinacalcet è stato, quindi, aggiunto il paracalcitolo $5 \mathrm{mcg} \times 3 /$ settimana per via endovenosa.

Due mesi più tardi il paziente ha sviluppato sulla mano sinistra, distalmente alla fistola artero-venosa, una lesione cutanea violacea evoluta successivamente in ulcerazione, necrosi e formazione di escara (Fig. 1); non sono stati notati segni clinici di ischemia né un'alterazione del deflusso venoso a carico della mano. La lesione è stata trattata con disinfezione, applicazione locale di collagenasi e antibiotici, senza alcun risultato favorevole. L'ecografia paratiroidea ha evidenziato al terzo inferiore della tiroide una formazione ipo-anecogena di $7 \mathrm{~mm}$. La biopsia cutanea ha rivelato, nel derma profondo, fenomeni di flogosi acuta microascessualizzante, una calcificazione delle pareti vasali, una necrosi ischemica sottocutanea e depositi di calcio. Nel Luglio del 2008, il paziente è stato sottoposto a una paratiroidectomia e i valori di PTH sono ritornati nella norma. Dopo la paratiroidectomia, la lesione cutanea è migliorata gradualmente fino alla completa guarigione.

\section{Discussione}

La calcifilassi viene riscontrata principalmente nei soggetti con patologie renali, ma, più raramente, anche in pazienti con normale funzione renale e neoplasie maligne, malattia epatica alcolica, connettivopatie, malattie infiammatorie intestinali e iperparatiroidismo primitivo. È stata osservata una prevalenza di CUA nel sesso femminile, verosimilmente per I'influenza patogenetica o predisponente dei fattori ormonali. L'incidenza di questa patologia è aumentata negli ultimi anni: la sua prevalenza è riportata nel $4 \%$ dei pazienti in dialisi. Nei soggetti in emodialisi con CUA viene riportata una sopravvivenza a 1 anno del $45.8 \%$, come risultato di infezioni locali o sistemiche e sepsi. MRC, diabete mellito, obesità e terapia con vitamina K-inibitori sono i principali fattori di rischio identificati. La calcifilassi è una vasculopatia ischemica a evoluzione obliterante, caratterizzata da calcificazioni vascolari, trombosi e lesioni necrotiche cutanee che evolvono in ulcere e nella formazione di escare. Le lesioni cutanee presentano caratteristiche cliniche peculiari in base al diverso pattern di coinvolgimento dei segmenti corporei interessati. La parete addominale, le cosce e la regione glutea sono le zone interessate nella maggioranza dei casi; questo coinvolgimento, definito "prossimale o centrale", sembra avere una prognosi peggiore rispetto a quello "distale o acrale", nel quale le lesioni si riscontrano agli arti inferiori. Nella review di Weenig, i soggetti con pattern di localizzazione distale hanno mostrato una sopravvivenza maggiore $(75.5 \%$ vs $26.2 \%)$ rispetto ai soggetti con localizzazione prossimale (5). Oltre alla cute, anche se eccezionalmente, altri organi e tessuti (polmoni, cuore, reni, tessuto muscolare striato, pancreas e apparato gastrointestinale) possono essere coinvolti. II ruolo della biopsia cutanea nel corso della calcifilassi è assai controverso a causa del potenziale rischio di necrosi nella sede del prelievo bioptico (6). Gli aspetti istologici della CUA sono suggestivi ma non patognomonici. La diagnosi differenziale comprende la malattia vascolare periferica, le vasculiti sistemiche, la sindrome embolica da colesterolo, il pioderma gangrenosum e la necrosi cutanea warfarin-indotta (7). La sua eziologia è multifattoriale. Le anomalie di laboratorio (iperparatiroidismo, iperfosforemia, ipercalcemia, elevato prodotto calciofosforo, deficit di inibitori delle calcificazioni vascolari) sono state implicate nella patogenesi di questa patologia. II ruolo dell'iperparatiroidismo secondario della MRC nella CUA non è ancora ben compreso. Un carattere peculiare della calcifilassi è la modificazione fenotipica delle cellule muscolari lisce in cellule simil-osteoidi. I miociti delle pareti vasali, esposti a elevate concentrazioni di fosfato inorganico, potrebbero essere trasformati in cellule simil-osteoblastiche nei pazien$t i$ in trattamento emodialitico con uno scarso controllo della fosforemia. In questo assetto metabolico, l'incremento della 
fosfatasi alcalina e l'espressione nelle cellule muscolari lisce delle pareti vasali di alcune proteine-marker del metabolismo osseo potrebbero indicarne la trasformazione osteogenica (8). Specifiche strategie terapeutiche (I'uso di dialisato a bassa concentrazione di calcio, la paratiroidectomia, i farmaci calciomimetici, i chelanti per il fosforo privi di calcio, i bifosfonati, l'ossigeno-terapia iperbarica e il tiosolfato sodico) sono limitate al report di case-series non controllate. Sinora nessuna terapia efficace è disponibile per il trattamento della CUA.

\section{Disclosures}

Financial support: No financial support was received for this submission.

Conflict of interest: The authors have no conflict of interest.

\section{Bibliografia}

1. Thongprayoon C, Cheungpasitporn W, Bruminhent J. Aggressive calciphylaxis in end-stage renal disease after a failed kidney allograft. Indian J Dermatol Venereol Leprol. 2015;81:50.

2. Marques SA, Kakuda AC, Mendaçolli TJ, Abbade LP, Marques
ME. Calciphylaxis: a rare but potentially fatal event of chronic kidney disease. Case report. An Bras Dermatol. 2013;88 (6 Suppl. 1):44-7.

3. Barbera V, Di Lullo L, Otranto G, Barbera G, Santoboni A. [Calciphylaxis: an enigma to the nephrologist]. G Ital Nefrol. 2012;29:674-82.

4. Verbeke F, Lindley E, Van Bortel L, et al. A European Renal Best Practice (ERBP) position statement on the Kidney Disease: Improving Global Outcomes (KDIGO) clinical practice guideline for the management of blood pressure in non-dialysisdependent chronic kidney disease: an endorsement with some caveats for real-life application. Nephrol Dial Transplant. 2014;29:490-6.

5. Weenig RH, Sewell LD, Davis MD, McCarthy JT, Pittelkow MR. Calciphylaxis: natural history, risk factor analysis, and outcome. J Am Acad Dermatol. 2007;56:569-79.

6. Latus J, Kimmel M, Ott G, Ting E, Alscher MD, Braun N. Early stages of calciphylaxis: are skin biopsies the answer? Case Rep Dermatol. 2011;3(3):210-5.

7. Wollina U. Update on cutaneous calciphylaxis. Indian J Dermatol. 2013;58(2):87-92.

8. Mizobuchi M, Finch JL, Martin DR, Slatopolsky E. Differential effects of vitamin $D$ receptor activators on vascular calcification in uremic rats. Kidney Int. 2007;72(6):709-15.

\section{TEST DI VERIFICA}

\section{La calcifilassi è:}
a) Una patologia autoimmune simile al LES
b) Una patologia sistemica a preminente manifestazio- ne cutanea
c) Una patologia infettiva batterica

\section{Nella calcifilassi si ritrovano:}
a) Ischemia cardiaca e cerebrale
b) Alterazioni epatiche metaboliche
c) Calcificazioni vascolari, trombosi e lesioni necrotiche cutanee

\section{Nella calcifilassi vengono interessati:}
a) I reni e l'apparato urinario
b) La fistola artero-venosa e le vene
c) Principalmente la cute ma anche altri organi

\section{Un evento non rilevato nella calcifilassi è:}
a) L'elevato rischio neoplastico
b) L'elevato rischio di infezioni
c) La modificazione delle cellule muscolari lisce in cel- lule simil-osteoidi

